\title{
Delegation and information revelation*
}

\author{
Axel Gautier ${ }^{\dagger} \quad$ Dimitri Paolini ${ }^{\ddagger}$
}

January 31, 2000

\begin{abstract}
This paper addresses the question of delegation in a principal-agent setting with asymmetric information. If the person who has the power to act, the principal, doesn't have the necessary information to make the best possible decision, she can address herself to someone, the agent, who has this information. Such delegation of authority has its drawbacks given that the agent may not implement the principal's ideal decision. Delegation is costly for the principal. This cost is called the loss of control. But delegation has also its benefits. We show that delegation is useful to reduce the initial asymmetry of information between the principal and the agent. The benefits of delegation are linked to the information transmitted by the agent to the principal. To show this, we model an organization composed of one principal and one agent. The organization should take a sequence of decisions that are affected by a common environemental parameter. We assume that there is an initial asymmetry of information between the principal and the subordinate agent: the agent knows the state of the world while the principal has only some prior about its distribution. Moreover, we assume that the principal cannot use revelation techniques à la Baron Myerson to elicit agent's superior information. In contrast, we adopt an incomplete contract framework and posit that the decision and the state of the world parameter cannot be contracted for. Therefore, the remaining contracting variable is the allocation of decision rights. With these simple contracts, we study how the agent's decision can signal his information to the principal. When the agent is in charge of a decision, his decision signals his information to the principal. The trade off
\end{abstract}

\footnotetext{
${ }^{*}$ We gratefully acknowledge F. Bloch, J. Crémer, seminars participants in IRES (Louvain-la-Neuve) and GREMAQ (Toulouse) for helpful comments and discussions. The usual disclaimer applies.

${ }^{\dagger}$ IRES and UCL Departement of economics Place Montesquieu, 3. 1348 Louvain la Neuve (Belgium) Email: gautier@ires.ucl.ac.be

${ }^{\ddagger}$ IRES and UCL Departement of economics Email: paolini@ires.ucl.ac.be
} 
between information transmitted through decisions under delegation and the associated loss of control is the heart of our analysis.

\section{Introduction}

This paper addresses the question of delegation in a principal-agent setting with asymmetric information. If the person who has the power to act, the principal $^{1}$, doesn't have the necessary knowledge (or information) to make the best possible decision, she can address herself to someone, the agent, who has this necessary information. Such delegation of authority has its drawbacks given that the agent may not necessarily implement the principal's ideal decision. In this paper,we suppose that the agent has preferences which are fixed but divergent from those of the principal. So delegation is costly for the principal. This cost is often referred in the literature as the loss of control. On the other hand, delegation has also its benefits. In a principal agent setting, the benefits of delegation are more difficult to identify $^{2}$ : for MMR, the benefits of delegation (they call them flexibility gains) are linked to the inability for the agents to communicate with the principal. For Laffont Martimort and Felli, a three layer hierarchy is better able to prevent collusion than a centralized structure. For Aghion and Tirole, delegating authority stimulates the effort of the agent in information acquisition. In this paper, we show that delegation may be useful to reduce the initial asymmetry of information between the principal and the agent. The benefits of delegation are linked to the information transmitted by the agent to the principal. The central result of the paper is to show that the delegated decisions are informative and the principal learns information when she delegates. To show this, we model an organization composed of one principal and one agent. The organization should take a sequence of (two) decisions that are affected by a common state of the world parameter. We assume that there is an initial asymmetry of information between the principal and

\footnotetext{
${ }^{1}$ We will refer as 'she' for the principal and 'he' for the agent.

${ }^{2}$ In the standard principal agent theory, following the revelation principle (Myerson, 1982), delegation is always weakly dominated by a grand contract between the principal and all the agents. To speak about delegation in a principal agent setting, one needs to relax some assumption of the revelation principle. Melumad, Mookherjee and Reichelstein (1992) (herafter MMR) relax the assumption of perfect communication between the principal and the agent, Felli relaxes the assumption of infinitely costly communication between agent, in order to allow collusion. Laffont and Martimort assume that communication between the principal and the agents is imperfect and that side contracting between agents is feasible. Aghion Tirole (1997) and this paper assume that the contracts are incomplete.
} 
the subordinate agent: the agent knows the state of the world parameter while the principal has only some prior about its distribution. Moreover, we assume that the principal cannot use revelation techniques à la Baron Myerson to elicit agent's superior information. In contrast, we adopt an incomplete contract framework (Grossman and Hart (1986), Hart and Moore (1990), Hart (1995) and Tirole (1999)) and posit that the decision and the state of the world parameter cannot be contracted for, neither ex ante nor ex post. Therefore, the remaining contracting variable is the allocation of decision rights. The only feasible contract is to decide who is in charge of each decision. Focusing on that simple contract is a convenient way to study how the agent's decision can signal his information to the principal. When the agent is in charge of a decision, his decision signals the state of the world to the principal. The trade off between information transmitted through decisions under delegation and the associated loss of control is the heart of our analysis. The first part of the paper studies how the (equilibrium) decisions of the agent reveals information to the principal. To show this, we use the properties of the signalling games. We show that to reveal his information, the agent may engage in costly signalling: i.e. take a decision which is not is preferred one. Using appropriate equilibrium refinement (Cho and Kreps' intuitive criterion) allow us to eliminate non informative equilibria. In other words, the only equilibrium that survives the intuitive criterion is the least costly separating equilibrium (Riley equilibrium). By using the intuitive criterion, we can say that the equilibrium described in the paper represents the upper limit of what could be achieve under delegation. After describing the outcomes under all the possible organizational structure ${ }^{3}$, we compute the costs and benefits associated with all the forms of delegation. We show that the benefits of delegation are linked to the asymmetry of information. On the other hand, the costs of delegation are function of the divergence of interests between the principal and the agent. With the help of these costs and benefits of delegation, we can compute the optimal organizational form. We can say (roughly) that delegation is preferred when agent's information has a great value and no delegation (a situation to which we refer as centralization) is preferred for large divergence of interest. We also show that the other forms of delegation like delegation of the second decision may emerge as the optimal organizational structure.

Another result is to show that, as the agent may not take his preferred decision in order to signal the information he posses, not only the importance

\footnotetext{
${ }^{3}$ We distinguish three forms of delegation: delegation of the first decision only, delegation of the second decision only and delegation of both decisions.
} 
of divergence of interest (the distance between the agent's and principal's ideal decisions) but also its direction (whether or not the principal prefers greater/smaller decisions than the agent) matters. To illustrate this point, consider the following example: if the agent takes a greater than his preferred decision under delegation in order to transmit the information he has about the state of the world to the principal, the utility of the principal is increased only if the principal's ideal decision is greater than those of the agent.

We believe that the trade off between information transmission and loss of control can be a rational for delegation in many complex organizations. The following three examples illustrates some of the relations, we would like to explain:

Example 1 (political decision): In the political area, decision makers (ministers, governments, ...) especially those who are new in office do not have the necessary knowledge of the problems and environment to take the best political decision. On the other hand, administrations, advisors and experts generally have this knowledge but lack of decision power. One solution for the minister is to delegate the decision to the administration. This has harmful effects if the administration has objectives that are not those of the minister (which seems to be plausible, as deciders changes while administration remains in place). Such a delegation has two advantages: first the administration is informed about (its) best decision and secondly, if the minister knows the administration's preference, he can learn through the observation of the decision what are the environmental conditions and use this information for subsequent decisions. The relations between ministers and administrations can be explained by a trade off between loss of control over decisions and information transfers.

Example 2 (vertical relations): In vertical relations, the client firm has to decide whether to buy a necessary component (delegation) or to produce it 'at home'. If the external supplier has a knowledge advantage (better knowledge of production process, patent, precise knowledge of cost conditions,...), outsourcing may be the good solution for the client. But the supplier may not act exactly in client's interests: the supplier may be reluctant to invest in specific assets (or specific knowledge), he may not engage some R\&D desired by the client,... But outsourcing has advantages as well as costs. If the client firm buys the component, there will be a transfer of knowledge from the supplying firm to the client. This transfer is such that, after buying, the client becomes more informed about the environment (the costs conditions or the technological possibilities for example). This informational benefits may be such that, in the future, the client firm decide to produce its own component. When the client initially outsources (delegates), even 
if the supplier will not act as a subsidiary firm would do, there is a transfer of knowledge from the supplier to the client. And this information can be used by the client for its subsequent decisions.

Example 3 (research): Many organizations such as firms, governments or international organizations delegate research to experts like consulting firms or universities while they have resources to produce it. We can explain such a delegation by the fact that even if the consulting firms and/or universities have a different objective from those of the government (for example valuable academic research vs support to decisions), they have a better knowledge of the 'state of research' in some particular field. Part of this expertise is transmitted through the research output and the deciders can use it as a basis for their subsequent research that can be oriented more toward support to decisions.

There are several papers related to ours. Aghion Tirole (1997, mentioned above), study the rational for delegation in a structure where the asymmetry of information between the principal and the agent is endogenous. They show that giving authority to the subordinate increases his incentive to be informed, which in turn increases his effective control over decisions (sometimes at the expense of the principal). The trade off studied by these authors is between loss of control and agent's increased initiative under delegation. Another paper that study the rational for delegation in an incomplete contract set up is Dessein (1999). He shows that the trade off between delegation and no delegation (where the agent only communicates some information to the principal) is a trade off between loss of control and loss of information. Under delegation, the decision is based on perfect information but take by an agent who doesn't share organization's preferences, while under no delegation, there is no bias in the decision but the information transmitted by the agent is noisy (à la Crawford Sobel): the principal doesn't learn the state of the world from the message transmitted by the agent but only improves her priors. In Legros (1993), at each period the principal delegates the choice of a policy to an agent with unknown preference. While taking a decision, the delegate trades off the immediate gain of taking his preferred decision (or a decision close to his preferred one) and the information about his preferences transmitted through the decision to the principal. This information is important because it affects the probability of being chosen as a delegate for the next period. Opposed to this paper, Legros shows that, when there is an asymmetry of information about preferences, the decisions cannot be completely informative and there is some bunching between types. Armstrong (1994) studies the problem of delegation when the principal ignores the state of the world and the agent's 
preference over decisions. His paper is not about the rational for delegation but to describe how to reduce the agent's discretionary power ( an approach similar to our second extension).

In the paper, we present two extensions of the model:

(i) We extend the analysis to allow for pre-play communication between the agent and the principal. More precisely, we describe when the agent communicates his information to the principal (not through decisions but through messages) when she retains the decisions rights over both decisions. We show that under some circumstances, the principal can achieve information transmission by the agent and keep control over both decisions. The existence of cheap talks, if it reduces the need for delegation, does not suppress it.

(ii) We try to see how the principal can limit the use (or abuse) of the decision right by the agent by imposing some rules that constraint the choice of the subordinate agent. We analyses rules that take the form of a limitation of the agents' subset of actions. In most case, a rule is useful tool to mitigate the losses of control but it has some limits. These limits are the requirement that the delegated decision remains informative (the principal should learn something by observing it) and that the rule doesn't constraint the agent to quit the organization (he must receive at least his reservation utility). Within these limits, we describe what is in our framework an optimal rule. Even if the principal can restrict agent's discretion, she cannot suppress all the costs associated with delegation.

The paper is organized as follow: in the next section, we present the model. In section 3, we describe the equilibrium decisions under the different organizational forms. We look, in section 4, at the costs and benefits of delegation. The model is extend to allow for cheap talk in section 5 . In section 6 , we describe how the principal may restrain the agent's discretionary power, and how this affects the outcome of the game. Section 7 concludes.

\section{Model}

We model an organization composed of one principal and one agent. This organization takes a sequence of two decisions (labelled $d_{1}$ and $d_{2}$ ) These decisions affect the welfare of both organization's members ${ }^{4}$. The utility of the principal and the agent are also affected by a common environmental

\footnotetext{
${ }^{4}$ Even if there is no dynamic in the model, we will sometimes refer to $d_{1}$ as the first period decision and $d_{2}$ as the second period decision.
} 
parameter $\theta$. This parameter is constant over periods ${ }^{5}$.

Contractual restrictions In this model, the only contracting variable is the allocation of decision rights over $d_{1}$ and $d_{2}$. These decision rights are allocated by the principal at the beginning of the first period either to herself or to the agent ${ }^{6}$. These contractual restrictions are consistent with the incomplete contract view of organizations. Giving authority to a subordinate agent is giving the right to select a decision from an allowed set (see Simon (1951), Grossman and Hart (1986), Hart and Moore (1990), Aghion and Tirole (1997)).

Environmental parameter We assume that the agent knows the "state of the world". This environmental parameter affects the utility of both the principal and the agent. The state of the world is drawn out of a set $\Theta$ from a common knowledge distribution $F(\theta)$. For simplicity, we assume ${ }^{7}$ that $\Theta=\left\{\theta_{1}, \theta_{2}\right\}$, with $\theta_{1}<\theta_{2}$ and we call $\Delta \theta=\theta_{2}-\theta_{1}$. The probability that $\theta$ equals $\theta_{1}$ is denoted $v_{1}$, the probability of $\theta=\theta_{2}$ equals $v_{2}=1-v_{1}$.

Decisions The choice of a decision represents the choice of a project implemented by the organization. The projects differs only in one dimension. We suppose that there is a continuum of possible decisions given by $] 0,+\infty[$.

Utility functions The utility of the agent is:

$$
U^{A}=\alpha_{1} d_{1}-\frac{\left(\theta-d_{1}\right)^{2}}{2}+\alpha_{2} d_{2}-\frac{\left(\theta-d_{2}\right)^{2}}{2}
$$

The utility of the principal is:

$$
U^{P}=\beta_{1} d_{1}-\frac{\left(\theta-d_{1}\right)^{2}}{2}+\beta_{2} d_{2}-\frac{\left(\theta-d_{2}\right)^{2}}{2}
$$

\footnotetext{
${ }^{5}$ This is a simplification. We can alternatively assume that the state of the world changes over periods and that there is some correlation between the state of the world in the two periods. In this case, the results of the paper remains qualitatively the same. The important assumption is that the observation of the first decision (under delegation) improves the information about the state of the world in the second period.

${ }^{6}$ The fact that the principal initially possess decision rights over both decisions can be justified by ownership of physical assets that confers the right to decide about their use or by institutional agreement, as it is the case in political decisions.

${ }^{7}$ The two states of the world framework simplifies the signalling game between, the principal and the agent without making it trivial.
} 
These utility functions exhibit three characteristics: first, the divergence of interest between the principal and the agent is measured by the different private benefit associated with each decision: $\alpha_{i} d_{i}$ and $\beta_{i} d_{i}, i=1,2$. These private benefits are measured in monetary units. . Second, the cost is state dependent and identical for the principal and the agent. The cost of implementing a decision $d_{i}$ in state $\theta$ is: $\frac{\left(\theta-d_{i}\right)^{2}}{2}$. Third, these functions are single peaked in each decision. The single peak assumption implies that the utility of the agent and the principal achieves a unique maximum in each decision for $d_{i}$ equals to respectively $\alpha_{i}+\theta$ and $\beta_{i}+\theta$. A high $\theta$ pushes up the ideal point of both the principal and the agent. So the interest of the two members are not completely antinomic.

The ratios $\frac{\alpha_{1}}{\alpha_{2}}$ and $\frac{\beta_{1}}{\beta_{2}}$ measure the relative importance of $d_{1}$ over $d_{2}$ for the agent and the principal ${ }^{8}$

The following picture represents the utility functions of the principal and the agent.

INSERT FIGURE 1 HERE

Note that these utility functions satisfies (trivially) the single crossing property.

Agent's participation: individual rationality After learning $\theta$ and the allocation of decision rights (the organizational form), the agent has the possibility of quitting the organization. We assume that the agent has an outside opportunity that gives him a utility level normalized to zero ${ }^{9}$. If the agent refuses to participate in the organization, it shuts down and both the principal and the agent get a zero payoff. A simple way to force the participation of the agent when $d_{1}$ and $d_{2}$ are such that $U^{A}\left(\theta, d_{1}, d_{2}\right)<0$ is to pay to the agent an unconditional wage $W$ such that: $U^{A}\left(\theta, d_{1}, d_{2}\right)+W=0$. In this case, only ex ante efficient organizations: organizations such that the total welfare (ex ante) is positive $\left(E U^{P}+U^{A} \geq 0\right)$, are carried out.

Timing of events The timing of decisions is as follow:

- The principal allocates decision rights.

- The agent observes the state of the world.

\footnotetext{
${ }^{8}$ Assuming that these ratios are different from one, helps us to identify more clearly in the analysis the influence of the first and the second decision.

${ }^{9}$ In the main course of this paper, we won't focus on individual rationality constraints. The exceptions are section $\mathrm{xx}$ and $\mathrm{xx}$ where it is shown that the participation constraints can affect the organizational choice.
} 
- The agent decides to stay within the organization or quit it.

- The first decision $d_{1}$ is taken

- The second decision $d_{2}$ is taken

- payoffs are collected

\section{Equilibrium decisions}

We assume that the only contracting variable is the allocation of decision rights over $d_{1}$ and $d_{2}$. There are four possible allocations of decisions right: centralization, delegation, complete delegation and second period delegation. We call centralization the case in which the principal keeps the decision rights over both decisions, delegation (or first period delegation) the case in which the better informed agent receives the decision right over $d_{1}$; complete delegation is the allocation of both decision rights to the agent and second period delegation is the allocation of $d_{1}$ to the principal and $d_{2}$ to the agent. This section describes the outcome of the game under these four organizational forms.

\subsection{Centralization}

Under centralization, the principal doesn't know the state of the world $\theta$ till the end of the game and the realization of costs. She therefore takes what we call "blind decisions": decisions that are not contingent on the value of $\theta$. These decisions are chosen in order to maximize the principal's expected utility and are given by the following equations:

$$
\begin{aligned}
& d_{1}=v_{1} \theta_{1}+v_{2} \theta_{2}+\beta_{1}=E \theta+\beta_{1} \\
& d_{2}=v_{1} \theta_{1}+v_{2} \theta_{2}+\beta_{2}=E \theta+\beta_{2}
\end{aligned}
$$

\subsection{Delegation}

When the principal delegates $d_{1}$ to the agent, she observes agent's decision before choosing $d_{2}$ This observation imposes a revision of her prior believes about the distribution of the state of the world parameter $\theta$. The game played by the principal and the agent is a standard signalling game. A Bayesian-Nash equilibrium of this signalling game is : $\left\{d_{1}^{*}\left(\theta_{1}\right), d_{1}^{*}\left(\theta_{2}\right), d_{2}^{*}\left(\theta_{1}\right), d_{2}^{*}\left(\theta_{2}\right), \mu\left(d_{1}\right)\right\}$ where $\mu\left(d_{1}\right)$ is the posterior distribution of $\theta$ after the principal has observed $d_{1}$. The posterior believes are computed with the Baye's rule. This kind 
of game usually has multiple equilibria ${ }^{10}$. We use the intuitive criterion (Cho-Kreps, 1987) to screen among all the possible equilibria. The use of the intuitive criterion can be justified by the following arguments: first, as we want to make comparisons between delegation and the other structure, the multiplicity of equilibria is a problem that can be solved by using this equilibrium refinement. Second, among the set of separating equilibria, the intuitive criterion selects the Pareto efficient one. This criterion not only get a rid of multiple equilibria but also have an efficiency content.

Proposition 1 The only equilibrium that survives the intuitive criterion is the least costly separating (LCS) equilibrium (Riley outcome).

This first proposition is the central result of the paper. It establishes that using the properties of signalling games, delegation is going together with a transfer of information from the agent to the principal. When the contracts are incomplete, the principal can still extract information from the agent by delegating the choice of some decision.

Corrolary 1 The LCS equilibrium is:

$$
d_{2}(\theta)=\beta_{2}+\theta
$$

$$
\text { If } \Delta \theta \geq\left|\alpha_{2}-\beta_{2}\right|
$$

$$
d_{1}(\theta)=\alpha_{1}+\theta
$$

$$
\text { If } \alpha_{2}-\beta_{2} \geq \Delta \theta
$$

$$
\begin{aligned}
& d_{1}\left(\theta_{1}\right)=\alpha_{1}+\theta_{1} \\
& d_{1}\left(\theta_{2}\right)=\alpha_{1}+\theta_{2}+\left(\sqrt{K_{1}}-\Delta \theta\right)
\end{aligned}
$$

Where $K_{1}=\left(2 \alpha_{2}-2 \beta_{2}-\Delta \theta\right) \Delta \theta$

$$
\begin{aligned}
& \text { If } \beta_{2}-\alpha_{2} \geq \Delta \theta \\
& \qquad \begin{array}{l}
d_{1}\left(\theta_{1}\right)=\alpha_{1}+\theta_{1}-\left(\sqrt{K_{2}}-\Delta \theta\right) \\
d_{1}\left(\theta_{2}\right)=\alpha_{1}+\theta_{2}
\end{array}
\end{aligned}
$$

Where $K_{2}=\left(2 \beta_{2}-2 \alpha_{2}-\Delta \theta\right) \Delta \theta$

\footnotetext{
${ }^{10}$ There are three kinds of equilibria: separating, pooling and semi pooling (or hybrid equilibria).
} 
We call the first case 'free lunch' or no costs signals. We refer to the other cases as the costly signaling cases. Now we prove proposition 1 and its corollary.

Proof. (i) Separating equilibria: The set of separating equilibria is the set of $\left\{d_{1}^{*}\left(\theta_{1}\right), d_{1}^{*}\left(\theta_{2}\right), d_{2}^{*}\left(\theta_{1}\right), d_{2}^{*}\left(\theta_{2}\right)\right\}$ that satisfy the following incentive compatible constraints:

$$
\begin{aligned}
& U^{A}\left(\theta_{1}, d_{1}^{*}\left(\theta_{1}\right), d_{2}^{*}\left(\theta_{1}\right)\right) \geq U^{A}\left(\theta_{1}, d_{1}^{*}\left(\theta_{2}\right), d_{2}^{*}\left(\theta_{2}\right)\right) \\
& U^{A}\left(\theta_{2}, d_{1}^{*}\left(\theta_{2}\right), d_{2}^{*}\left(\theta_{2}\right)\right) \geq U^{A}\left(\theta_{2}, d_{1}^{*}\left(\theta_{1}\right), d_{2}^{*}\left(\theta_{1}\right)\right)
\end{aligned}
$$

In a separating equilibrium, believes are: $\mu\left(\theta_{1} \mid d_{1}^{*}\left(\theta_{1}\right)\right)=1, \mu\left(\theta_{1} \mid d_{1}^{*}\left(\theta_{2}\right)\right)=$ 0 . With these believes we can compute $d_{2}^{*}(\theta)$ :

$$
d_{2}^{*}(\theta)=\beta_{2}+\theta
$$

Using (xx) and the definition of $U^{A}$, the constraint $I C_{1}$ and $I C_{2}$ become:

$$
\left(\alpha_{1}+\theta_{1}\right)\left(d_{1}^{*}\left(\theta_{1}\right)-d_{1}^{*}\left(\theta_{2}\right)\right)+\frac{1}{2}\left(d_{1}^{*}\left(\theta_{2}\right)^{2}-d_{1}^{*}\left(\theta_{1}\right)^{2}\right) \geq \Delta \theta\left(\alpha_{2}-\beta_{2}-\frac{\Delta \theta}{2}\right)
$$

$\mathrm{IC} \prime_{1}(10)$

$$
\left(\alpha_{1}+\theta_{2}\right)\left(d_{1}^{*}\left(\theta_{2}\right)-d_{1}^{*}\left(\theta_{1}\right)\right)+\frac{1}{2}\left(d_{1}^{*}\left(\theta_{1}\right)^{2}-d_{1}^{*}\left(\theta_{2}\right)^{2}\right) \geq \Delta \theta\left(\beta_{2}-\alpha_{2}-\frac{\Delta \theta}{2}\right)
$$

$\mathrm{IC}_{2}(11)$

To characterize the separating equilibrium, we have to identify the relevant incentive constraint. The RHS of $I C{ }_{i}$ represents the benefits for type $\theta_{i}$ of mimicking the type $\theta_{j} ; i, j=1,2$. We identify 3 cases: case S.1: the RHS of $I C \prime_{1}$ is positive $\left(\alpha_{2}-\beta_{2}-\frac{\Delta \theta}{2} \geq 0\right)$, in this case, the utility of $\theta_{1}$ increases if he acts as $\theta_{2}$. Case S.2: The RHS of $I C \iota_{2}$ is positive, in this case, the utility of $\theta_{2}$ increases if he acts as $\theta_{1}$. Case S.3: Both RHS are negative which means that no type has an incentive to misrepresent his type ${ }^{11}$.

Case S.1: Suppose that $\alpha_{2}-\beta_{2}-\frac{\Delta \theta}{2} \geq 0$. The set of separating equilibrium is:

$$
\begin{aligned}
& d_{1}^{*}\left(\theta_{1}\right)=\alpha_{1}+\theta_{1} \\
& d_{1}^{*}\left(\theta_{2}\right) \in D \equiv\left\{d_{1}\left(\theta_{2}\right) \mid I C \prime_{1}, I R_{1}\right\}
\end{aligned}
$$

\footnotetext{
${ }^{11}$ It is impossible to have the two incentive contraint relevant at the same time. This comes from the single crossing property of the utility function.
} 
The set $D$ is the set of decisions that satisfy the participation constraint for type $\theta_{2}$ and the constraint $I C \prime_{1}$. Using (xx), and the definition of the utility function, $D \equiv D_{1} \cap D_{2}$. $D_{1}$ is the set of incentive compatible decisions: $\left.\left.D_{1} \equiv\right] 0, \alpha_{1}+\theta_{1}-\sqrt{K_{1}}\right] \cup\left[\alpha_{1}+\theta_{1}+\sqrt{K_{1}},+\infty\left[\right.\right.$. and $D_{2}$ is the set of decisions that left a positive utility to agent $\theta_{2}: D_{2} \equiv\left[\alpha_{1}+\theta_{2}-\sqrt{K_{3}}, \alpha_{1}+\theta_{2}+\sqrt{K_{3}}\right]$ where $K_{3}=\left(\alpha_{1}+\theta_{2}\right)^{2}-\beta_{2}^{2}+\frac{\alpha_{2} \beta_{2}}{2}-\theta_{2}^{2}$.

This equilibrium is supported by pessimistic believes: $\mu\left(\theta_{1} \mid d_{1}\right)=1, \forall d_{1} \neq$ $d_{1}^{*}\left(\theta_{2}\right)$ and $\mu\left(\theta_{1} \mid d_{1}\right)=0$, if $d_{1}=d_{1}^{*}\left(\theta_{2}\right)$.

Now we us the intuitive criterion to eliminate all the equilibria but the LCS one.

An equilibrium is not intuitive if:

$$
\exists d_{1}\left(\theta_{2}\right) \in \text { Ds.t. } U^{A}\left(\theta_{2}, d_{1}\left(\theta_{2}\right)\right)>U^{A}\left(\theta_{2}, d_{1}^{*}\left(\theta_{2}\right)\right)
$$

The idea behind this condition is if the principal observes a deviation from the equilibrium decision $d_{1}^{*}\left(\theta_{2}\right)$ to an other incentive compatible decision $d_{1}\left(\theta_{2}\right) \in D$, it can be done only by the type $\theta_{2}$. Observing such an out-of-equilibrium action, the principal has to update her believes about $\theta$ to: $\mu\left(\theta_{1} \mid d_{1}\left(\theta_{2}\right)\right)=0$.

It is easy to show that such the only surviving equilibrium is: $d_{1}^{*}\left(\theta_{2}\right)=$ $\alpha_{1}+\theta_{2}$ if $\alpha_{2}-\beta_{2} \geq \Delta \theta$ and $d_{1}^{*}\left(\theta_{2}\right)=\alpha_{1}+\theta_{1}+\sqrt{K_{1}}=\alpha_{1}+\theta_{2}+\left(\sqrt{K_{1}}-\Delta \theta\right)$ otherwise. The first corresponds to the case where $\alpha_{1}+\theta_{2} \in D^{12}$. In the second case, $d_{1}^{*}\left(\theta_{2}\right)$ is the decision closest to $\alpha_{1}+\theta_{2}$ within $D$.

Case S.2 and S.3 are similar to this case.

(ii) Pooling equilibria

A pooling equilibrium is defined as: $d_{1}^{*}\left(\theta_{1}\right)=d_{1}^{*}=d_{1}^{*}\left(\theta_{2}\right), d_{2}^{*}=E \theta+\beta_{2}$, $\mu\left(\theta_{1} \mid d_{1}\right)=v_{1}$. To define the set of pooling equilibria, we have to define out-of-equilibrium believes that support the equilibrium. First, we have to identify which type of agent wants to quit the pooling equilibrium. An agent prefers to quit the pooling equilibrium, if his utility is greater if he can signal his type. Again, we distinguish three cases: Case P.1: $\left(\alpha_{2}-\beta_{2}\right) \geq \frac{v_{2} \Delta \theta}{2}$ this case corresponds to the case where $\theta_{2}$ prefers $d_{2}\left(\theta_{2}\right)=\beta_{2}+\theta_{2}$ rather than $d_{2}(\theta)=E \theta+\beta_{2}$. The agent $\theta_{2}$ has in this case an interest to signal his type. Case P.2: $\left(\beta_{2}-\alpha_{2}\right) \geq \frac{v_{1} \Delta \theta}{2}$ This is the opposite case where the utility of agent $\theta_{1}$ decreases if he cannot signal his type. Case P.3: Both types prefer if they can signal their type ${ }^{13}$.

\footnotetext{
${ }^{12}$ Notice that $\alpha_{1}+\theta_{2} \in D$ if the costs of mimicking $\theta_{2}$ for $\theta_{1}$ (which are the lost utility when $\theta_{1}$ chooses $d_{1}\left(\theta_{1}\right)=\alpha_{1}+\theta_{2}$ instead of $\left.d_{1}\left(\theta_{1}\right)=\alpha_{1}+\theta_{2}\right)$ are greater than the benefits given by the RHS of $I C_{1}^{\prime}$. These costs of mimicking are $\frac{\Delta \theta^{2}}{2}$, and they are greater than benefits if $\alpha_{2}-\beta_{2} \leq \Delta \theta$.

${ }^{13}$ This is a consequence of the single crossing condition.
} 
Case P.1: In this case, as $\theta_{2}$ wants to signal his type, the pooling equilibrium $d_{1}^{*}, d_{2}^{*}=E \theta+\beta_{2}$ is supported by believes: $\mu\left(\theta_{1} \mid d_{1}\right)=1$ if $d_{1} \neq d_{1}^{*}$ and $\mu\left(\theta_{1} \mid d_{1}\right)=v_{1}$ if $d_{1}=d_{1}^{*}$ To be an equilibrium, $d_{1}^{*}, d_{2}^{*}$ must satisfy the following individual rationality constraints $U^{A}\left(\theta_{i}, d_{1}^{*}, d_{2}^{*}\right) \geq 0, i=1,2$. We call $D_{I R}$ the set of $d_{1}^{*}$ for which individual rationality is satisfied. Moreover, the two following equilibrium requirement must be satisfied:

$$
\begin{aligned}
& U^{A}\left(\theta_{1}, d_{1}^{*}, d_{2}^{*}\right) \geq U^{A}\left(\theta_{1}, d_{1}, d_{2}=\theta_{1}+\beta_{2}\right) \forall d_{1} \neq d_{1}^{*} \\
& U^{A}\left(\theta_{2}, d_{1}^{*}, d_{2}^{*}\right) \geq U^{A}\left(\theta_{2}, d_{1}, d_{2}=\theta_{1}+\beta_{2}\right) \forall d_{1} \neq d_{1}^{*}
\end{aligned}
$$

Using these two conditions, we can define the set $D$ of pooling equilibria. The condition $\mathrm{xx}$ is satisfied for all $d_{1}$ if it is satisfied for $d_{1}=\alpha_{1}+\theta_{1}$. Condition xx resumes to: $d_{1}^{*} \in D_{1} \equiv\left[\alpha_{1}+\theta_{1}-\sqrt{A}, \alpha_{1}+\theta_{1}+\sqrt{A}\right]$, where $A=2 v_{2} \Delta \theta\left(\alpha_{2}-\beta_{2}-\Delta \theta\right)$. Condition xx is satisfied for all $d_{1}$ if it is satisfied for $d_{1}=\alpha_{1}+\theta_{2} \quad \mathrm{xx}$ becomes: $d_{1}^{*} \in D_{2} \equiv\left[\alpha_{1}+\theta_{2}-\sqrt{B}, \alpha_{1}+\theta_{2}+\sqrt{B}\right]$, where $B=2 v_{2} \Delta \theta\left(\alpha_{2}-\beta_{2}\right)+\left(1-v_{1}^{2}\right) \Delta \theta$. The set of pooling equilibria is defined as: $d_{1}^{*} \in D \equiv D_{1} \cap D_{2} \cap D_{I R}$.

Suppose that set is non empty, we use the intuitive criterion to suppress all the pooling equilibria.

add some stuff here

The cases P.2 and P.3 are similar. To eliminate hybrid equilibria, we proceed as in the case of pooling equilibria.

Q.E.D

Proposition 1 establishes that delegating $d_{1}$ suppress the asymmetric information between the principal and the agent. In the next section, we show that such a delegation has benefits as well as costs and that even if it reduces the information asymmetry it is not always optimal to delegate.

\subsection{Complete delegation and second period delegation}

Finally, we mention the two other possible allocations of decision rights: the complete delegation and the second period delegation. These cases have in common that there is no problem of information transmission from the agent to the principal. Under complete delegation, the agent takes his preferred decisions $d_{1}$ and $d_{2}$ :

$$
\begin{aligned}
& d_{1}=\alpha_{1}+\theta \\
& d_{2}=\alpha_{2}+\theta
\end{aligned}
$$

The complete delegation of decision rights to the agent raises a problem of time consistency: after observing $d_{1}$, the principal has an incentive to 
retake from the agent the control right over $d_{2}$. Indeed, after observing $d_{1}$, the principal learns the state of the world $\theta$. Delegating the second period decision has no benefit but just a cost ${ }^{14}$. Therefore, if the principal cannot commit to the allocation of decision right over $d_{2}$ to the agent ${ }^{15}$, he will anticipate that the allocation of decision will be changed. If there is no commitment to the allocation of the second decision, the case of complete delegation is identical to the case of delegation ${ }^{16}$.

If the principal delegates only $d_{2}$, she takes $d_{1}$ according to (1) and the agent takes $d_{2}$ according to (10). In this case, only the second decision is taken by an informed party. Second period delegation is equivalent to a one period model where information transmission plays no role.

\section{Costs and benefits of delegation}

When the principal delegates some decision to the agent, she suffers a loss of control because the agent doesn't have the same preferences over decisions. But, on the other hand, the agent is better informed about the state of the world and delegated decisions are token on the base of a better information. Moreover, when the principal delegates $d_{1}$, there is a transfer of information from the agent to the principal (proposition 1). Delegation has a benefit as well as a cost. The benefits are linked to the information, the cost to the divergence of interests. We call the latter costs of delegation and the former benefits of information. In this section, we provide a convenient way to isolate costs and benefits associated with delegation. Using these definitions, it is easy to identify the optimal organization.

\subsection{Benefits of delegation (benefits of information)}

What are the benefits of delegation? What kind of advantages can the principal have when she delegates some decisions to the agent? Obviously,

\footnotetext{
${ }^{14}$ Except if the interests over $d_{2}$ are perfectly congruent.

${ }^{15}$ Aghion and Tirole (1997) study this particular problem of commitment to a given organizational structure. They study how the principal can credibly commit not to infer too much in agent's decision in order to stimulate his initiative. They distinguish formal and real authority: which are right to decide versus effective control over decisions. A credible way for the principal to not interfer in agent's decisions is to give formal authority to the agent. But, even in the case where the principal keeps formal autorithy, the principal can stimulate the initiative of the agent by using costly monitoring technology or by monitoring a large number of agents and be in a situation of overload.

${ }^{16}$ For the remaining of the paper, when we speak about complete delegation, we assume that the principal can commit to a given allocation of decision rights.
} 
these advantages come from the informational structure of the model. As the agent has superior information, under delegation, decisions are based on the actual value of $\theta$ rather than on its prior distribution. Therefore, the benefits of delegation are the benefits of having an informed decider. The benefits of information can be easily computed by taking the difference between the expected utility of an informed principal and the expected utility of an uninformed principal. In both cases, we suppose that the principal takes both decisions. When she is informed, $d_{i}=\beta_{i}+\theta ; i=1,2$, when she's not, $d_{i}=\beta_{i}+v_{1} \theta_{1}+v_{2} \theta_{2} ; i=1,2$. The benefits of delegation are the difference $E U^{P}$ (Principal informed $)-E U^{P}$ (Centralization) $=v_{1} v_{2} \Delta \theta^{2}$. Being informed increases $E U^{P}$ by $v_{1} v_{2} \Delta \theta^{2}$. We can decompose this benefits in two parts: the benefits of an informed decision $d_{1}$ and the benefits of an informed decision $d_{2}$. By symmetry of the model, the per decision benefits of information is equal to: $\frac{v_{1} v_{2} \Delta \theta^{2}}{2}$. The following lemma follows immediately:

Lemma 1 The benefits of information are equal to:

$$
v_{1} v_{2} \Delta \theta^{2}
$$

when the principal delegates $d_{1}$ or $d_{1}$ and $d_{2}$.

They are equal to:

$$
\frac{v_{1} v_{2} \Delta \theta^{2}}{2}
$$

when the principal delegates $d_{2}$.

When the principal delegates $d_{1}$, both decisions are taken on the basis of the true value of $\theta^{17}$ because the first decision signals $\theta$ to the principal and she becomes informed before choosing $d_{2}$ as established in proposition 1. While under delegation of $d_{2}$ only, the first decision is not taken under complete information but only on the base of priors. These benefits are half of what they are under complete or first period delegation.

The positive benefits of information reflects the quadratic nature of the cost function used in the model. These benefits are a quadratic function of $\Delta \theta$. When $\Delta \theta$ is large, agent's information has a great value.

\subsection{Costs of delegation (loss of control)}

To compute the benefits of delegation, we have abstract from the loss of control associated with diverging interests between the principal and the

\footnotetext{
${ }^{17}$ This is, by definition, also the case under complete delegation
} 
agent. Now, similarly, to compute the costs of delegation we abstract from the asymmetry of information between the principal and the agent and concentrate only on the problem of diverging interests. The costs of delegation or loss of control are related to the fact that the agent doesn't share the principal's preferences over decisions. So these costs are function of the divergence of interest between the principal and the agent. To measure them, we compare the principal's expected utility in two situations: in the first, the principal is informed and take all the decisions, in the second, she's informed and delegates one or two decisions to the agent. $C D_{i}=$ $E U^{P}$ (Principal informed $)-E U^{P}$ (Delegation of $\left.d_{i}\right)$. By doing so, we abstract from informational gains that can be produced by delegation

Lemma 2 The costs of first period delegation $\left(C D_{1}\right)$ are:

$$
\text { If } \Delta \theta \geq\left|\alpha_{2}-\beta_{2}\right| \text {, }
$$

$$
C D_{1}=\frac{\left(\alpha_{1}-\beta_{1}\right)^{2}}{2}
$$

If $\alpha_{2}-\beta_{2} \geq \Delta \theta$,

$$
C D_{1}=\frac{\left(\alpha_{1}-\beta_{1}\right)^{2}}{2}+v_{2}\left(\sqrt{K_{1}}-\Delta \theta\right)\left(\alpha_{1}-\beta_{1}+\frac{\sqrt{K_{1}}-\Delta \theta}{2}\right)
$$

If $\beta_{2}-\alpha_{2} \geq \Delta \theta$,

$$
C D_{1}=\frac{\left(\alpha_{1}-\beta_{1}\right)^{2}}{2}+v_{1}\left(\sqrt{K_{2}}-\Delta \theta\right)\left(\beta_{1}-\alpha_{1}+\frac{\sqrt{K_{2}}-\Delta \theta}{2}\right)
$$

The costs of second period delegation $\left(C D_{2}\right)$ are given by:

$$
C D_{2}=\frac{\left(\alpha_{2}-\beta_{2}\right)^{2}}{2}
$$

The costs of complete delegation $\left(C D_{12}\right)$ are given by:

$$
C D_{12}=\frac{\left(\alpha_{1}-\beta_{1}\right)^{2}}{2}+\frac{\left(\alpha_{2}-\beta_{2}\right)^{2}}{2}
$$

The lemma shows that the costs of delegation are an increasing function of the distance between the principal's and agent's ideal decision. $C D_{2}$ is a function of the distance between $\alpha_{2}$ and $\beta_{2}, C D_{12}$ is a function of the distance between $\alpha_{1}$ and $\beta_{1}$ and $\alpha_{2}$ and $\beta_{2}$. Under first period delegation, $C D_{1}$ can take three values depending on the signalling equilibrium described in our first corollary. In the case of free lunch signals, the costs of delegation are simply a function of the distance between $\alpha_{1}$ and $\beta_{1}$. The greater the 
distance between the principal's and the agent's ideal point, the greater $C D_{1}$ is. An interesting question is to see if the costs of delegation are reduced or increased when the signals are costly for the agent. This depends on (i) the direction and (ii) the size of the change in the decision relative to the case of free lunch signals. Take the case where the agent sends a costly signal in state $\theta_{2}{ }^{18}$ : it implies that $d_{1}\left(\theta_{2}\right)=\alpha_{1}+\theta_{2}+\sqrt{K_{1}}-\Delta \theta>\alpha_{1}+\theta_{2}$. This increases in $d_{1}\left(\theta_{2}\right)$ benefits to the principal if (i) $\beta_{1}>\alpha_{1}$ which means that the principal's ideal point is greater than those of the agent and (ii) the increase in $d_{1}\left(\theta_{2}\right)$ is not too big relative to $\beta_{1}-\alpha_{1}$ : when the agent takes $d_{1}\left(\theta_{2}\right)=\alpha_{1}+\theta_{2}$, the distance between the principal's ideal point and the actual decision is $\left(\beta_{1}-\alpha_{1}\right)$. The utility of the principal will be increased in state $\theta_{2}$ if the distance between the decision $d_{1}\left(\theta_{2}\right)=\alpha_{1}+\theta_{2}+\sqrt{K_{1}}-\Delta \theta$ and her ideal point $\beta_{1}+\theta_{2}$ is smaller than $\left(\beta_{1}-\alpha_{1}\right)$. Because of the single peakness assumption, the maximal increase $\sqrt{K_{1}}-\Delta \theta$ that left a positive utility gain to the principal is twice the distance $\beta_{1}-\alpha_{1}$. The figure 2 summarizes the argument presented here.

INSERT FIGURE 2 HERE

¿From the previous discussion, we can establish the following proposition:

Proposition 2 (i) When $\Delta \theta$ is small $\left(\Delta \theta \leq\left|\alpha_{2}-\beta_{2}\right|\right.$, the costs of first period delegation are reduced if:

When $\alpha_{2}-\beta_{2} \geq \Delta \theta$, (i) $\beta_{1}>\alpha_{1}$ and (ii) $2\left(\beta_{1}-\alpha_{1}\right)>\left(\sqrt{K_{1}}-\Delta \theta\right)$

When $\beta_{2}-\alpha_{2} \geq \Delta \theta$, (i) $\alpha_{1}>\beta_{1}$ and (ii) $2\left(\alpha_{1}-\beta_{1}\right)>\left(\sqrt{K_{2}}-\Delta \theta\right)$

(ii) When $\alpha_{1}=\alpha_{2}$ and $\beta_{1}=\beta_{2}$, the costs of delegation always increase.

This proposition establishes that not only the distance between the principal's and agents ideal points matters but also the direction of preferences. We will see that the costly signals creates an asymmetry in the choice of the organizational structure. For a given $\left|\alpha_{1}-\beta_{1}\right|$, the choice will differ in the case $\alpha_{1} \geq \beta_{1}$ from the case $\beta_{1} \geq \alpha_{1}$.

\subsection{Optimal organizational structure}

¿From the computation of costs and benefits of delegation, comparisons between the four organizational structure are easy. Using lemma 1 and 2, the next proposition is immediate:

${ }^{18}$ This corresponds to the case where $\alpha_{2}-\beta_{2} \geq \Delta \theta$ 
Proposition 3 The optimal organizational structure is the organization for which the difference between the associated costs and benefits is maximal with the costs and benefits of centralization both equal to zero.

The following technical lemmas give the optimal organizational structure. Our results are summarized in propositions $\mathrm{xx}, \mathrm{xx}$ and $\mathrm{xx}$ as well as in figure3.

Lemma 3 The optimal organization is:

to delegate $d_{1}$ if:

$$
C D_{1} \leq v_{1} v_{2} \Delta \theta^{2}
$$

and if $C D_{2} \leq \frac{v_{1} v_{2} \Delta \theta^{2}}{2}$ :

$$
\frac{\left(\alpha_{1}-\beta_{1}\right)^{2}}{2}-\frac{\left(\alpha_{2}-\beta_{2}\right)^{2}}{2} \leq \frac{v_{1} v_{2} \Delta \theta^{2}}{2}
$$

to delegate $d_{2}$ if:

$$
C D_{2} \leq \frac{v_{1} v_{2} \Delta \theta^{2}}{2}
$$

or

$$
\frac{\left(\alpha_{1}-\beta_{1}\right)^{2}}{2}-\frac{\left(\alpha_{2}-\beta_{2}\right)^{2}}{2} \geq \frac{v_{1} v_{2} \Delta \theta^{2}}{2}
$$

and centralization otherwise.

Proof. We first show that complete delegation is always dominated: when $\Delta \theta \geq\left|\alpha_{2}-\beta_{2}\right|, C D_{1}=\frac{\left(\alpha_{1}-\beta_{1}\right)^{2}}{2}$ is always smaller than $C D_{12}$. Therefore if complete delegation dominates centralization $\left(C D_{12} \leq v_{1} v_{2} \Delta \theta^{2}\right)$, complete delegation is dominated by first period delegation (by proposition $\mathrm{xx}$ ). When $\Delta \theta \leq\left|\alpha_{2}-\beta_{2}\right|$, it implies $\Delta \theta^{2} \leq\left(\alpha_{2}-\beta_{2}\right)^{2}$. Then $\frac{\left(\alpha_{1}-\beta_{1}\right)^{2}}{2}+\frac{\left(\alpha_{2}-\beta_{2}\right)^{2}}{2}$ cannot be smaller than $v_{1} v_{2} \Delta \theta^{2}$, which means that centralization dominates complete delegation.

First period delegation dominates when $C D_{1} \geq v_{1} v_{2} \Delta \theta^{2}$, and if second period delegation dominates centralization, $C D_{1}-v_{1} v_{2} \Delta \theta^{2}$ must be greater than $C D_{2}-\frac{v_{1} v_{2} \Delta \theta^{2}}{2}$. If this last expression is positive, it implies $\Delta \theta \geq$ $\left|\alpha_{2}-\beta_{2}\right|$ and we have $C D_{1}=\frac{\left(\alpha_{1}-\beta_{1}\right)^{2}}{2}$. Equation 21 is simply a reformulation of these conditions.

Second period delegation dominates if $C D_{2} \leq \frac{v_{1} v_{2} \Delta \theta^{2}}{2}$ (equation 22) and if $\frac{v_{1} v_{2} \Delta \theta^{2}}{2}-C D_{2} \geq v_{1} v_{2} \Delta \theta^{2}-C D_{1}$ (equation 23).

Q.E.D 
Proposition 4 For large $\Delta \theta$, some form of delegation is optimal. If $\mid \alpha_{1}-$ $\beta_{1} \mid$ is large compared to $\left|\alpha_{2}-\beta_{2}\right|$, the principal delegates $d_{2}$ only, otherwise she delegates $d_{1}$.

When $\Delta \theta$ is large, the agent's information has a great value. It is important for the principal to have informed decisions which imply that delegation is optimal. She delegates $d_{2}$ in the case where the costs of delegating $d_{1}$ is large relative to the costs of delegating $d_{2}$. Notice also that when $\Delta \theta$ is large, the agent's can transfer their information at no cost.

Proposition 5 For small $\Delta \theta$, the optimal organizational structure is either to delegate $d_{1}$ or centralization. The choice depends on (i) the distance between $\alpha_{1}$ and $\beta_{1}$ and (ii) the sign of the difference $\left(\alpha_{1}-\beta_{1}\right)$.

When $\Delta \theta$ is small, it is more difficult for the agent to transfer his information to the principal. This difficulty leads to more extreme decisions than in the case of a high $\Delta \theta$. More extreme decisions benefits to the principal only if, an informed principal would have been more extreme than the agent. This explains why when for a given $\left|\alpha_{1}-\beta_{1}\right|$, when the interests go in the same direction, the principal delegates more often and when they go in opposite direction, she delegates less.

Proposition 6 When $\alpha_{1}=\alpha_{2}$ and $\beta_{1}=\beta_{2}$, the optimal organizational structure is delegation if $C D_{1} \leq v_{1} v_{2} \Delta \theta^{2}$ and centralization otherwise.

If both decisions have the same importance, when the principal delegates, she delegates $d_{1}$.

The following figure (INSERT FIGURE 3 HERE) represents optimal organizational structure(centralization vs delegation) for the case of $\alpha_{2}>\beta_{2}$. As we can see, an increase in $\Delta \theta$ affects the organizational structure in a twofold way: first it increases the benefits of information (favors delegation) and second, it affects the agent's decision. A high $\Delta \theta$ favors free lunch signals instead of costly signals, and this in turn affect the organizational choice.

\section{$5 \quad$ Cheap talks}

Without modifying the assumptions on contract, we can identify circumstances under which the principal never delegates and still achieve information revelation. A situation where the principal enjoys the benefits of 
information without incurring any costs of delegation. This will be the case if, when the principal retains decision rights over $d_{1}$ and $d_{2}$, the agent communicates freely and truthfully the state of the world to the principal ${ }^{19}$. We call this case cheap talks. If the principal is informed by the agent, she takes the decisions $d_{1}$ and $d_{2}$ that corresponds to her ideal point $\beta_{i}+\theta ; i=1,2$. The agents communicate the relevant information to the principal if the following sets of conditions are satisfied: the first set of conditions (equations 15 and 16) ensures that the agent prefers an informed principal to centralization. The second set (equation 19 and 20) ensures that the agent communicates a different information in state $\theta_{1}$ than in state $\theta_{2}$.

$$
\begin{aligned}
& U^{A}\left(\theta_{1}, d_{i}=\beta_{i}+\theta_{1}, i=1,2\right) \geq U^{A}\left(\theta_{1}, d_{i}=\beta_{i}+v_{1} \theta_{1}+v_{2} \theta_{2}, i=1,2\right) \\
& U^{A}\left(\theta_{2}, d_{i}=\beta_{i}+\theta_{2}, i=1,2\right) \geq U^{A}\left(\theta_{2}, d_{i}=\beta_{i}+v_{1} \theta_{1}+v_{2} \theta_{2}, i=1,2\right)
\end{aligned}
$$

If these conditions are both satisfied, the agent always prefers an informed principal to centralization. If one of these conditions doesn't hold, both agents will send the same message and the principal won't learn the state of the world (unless she delegates). Using the definition of $U^{A},(15)$ and (16) can be rewritten as:

$$
\begin{aligned}
& \alpha_{1}+\alpha_{2}-\beta_{1}-\beta_{2} \leq v_{2} \Delta \theta \\
& \beta_{1}+\beta_{2}-\alpha_{1}-\alpha_{2} \leq v_{1} \Delta \theta
\end{aligned}
$$

And it is clear that at least one of these conditions is trivially satisfied.

The agent communicates a different message in state $\theta_{1}$ than in state $\theta_{2}$ if the following conditions are both satisfied (or both unsatisfied):

$$
\begin{aligned}
& U^{A}\left(\theta_{1}, d_{i}=\beta_{i}+\theta_{1}, i=1,2\right) \geq U^{A}\left(\theta_{1}, d_{i}=\beta_{i}+\theta_{2}, i=1,2\right) \\
& U^{A}\left(\theta_{2}, d_{i}=\beta_{i}+\theta_{2}, i=1,2\right) \geq U^{A}\left(\theta_{2}, d_{i}=\beta_{i}+\theta_{1}, i=1,2\right)
\end{aligned}
$$

Using the definition of $U^{A}$, we have:

$$
\begin{aligned}
& \alpha_{1}+\alpha_{2}-\beta_{1}-\beta_{2} \leq \Delta \theta \\
& \beta_{1}+\beta_{2}-\alpha_{1}-\alpha_{2} \leq \Delta \theta
\end{aligned}
$$

These conditions are weaker than the conditions (17) and (18). The agent communicates $\theta$ to the principal only if he prefers an informed principal to centralization.

\footnotetext{
${ }^{19}$ Or any pair of messages $m\left(\theta_{1}\right), m\left(\theta_{2}\right)$ from which the principal can infer the state of the world.
} 
Proposition 7 If $\alpha_{1}+\alpha_{2}-\beta_{1}-\beta_{2} \leq v_{2} \Delta \theta$ and $\beta_{1}+\beta_{2}-\alpha_{1}-\alpha_{2} \leq v_{1} \Delta \theta$, the optimal organization is centralization and the agent communicates his private information to the principal.

This proposition describes the case in which retaining the information hurts the agent. From this discussion, it appears that communication is an important factor in the choice of an organizational form. The following figure (INSERT FIGURE HERE) shows when cheap talks and centralization dominates delegation.

\section{Rules}

When the principal left some power to the agent, she would like to reduce the discretion of the agent by imposing some constraints on the choice of the subordinate agent. Constraining the choice of the agent appears to be a useful way to reduce the cost of delegation while preserving what we called the benefits of information. To reduce the discretion of the agent, the principal may constraint the agent to choose $d_{1}$ within a given subset $L$. By doing so, we will say that the principal imposes a rule that limits the discretion of the agent in the choice of $d_{1}$. We define a rule as a compulsory requirement that must be followed when the principal delegates the decision rights over $d_{1}$. Our interest in this subsection is to see how the principal can effectively reduces the cost of delegation by imposing such a rule and to compute the optimal way of doing so, what we call the optimal rule. The emergence of rule that lower the power of incentives is a fundamental characteristic of any organization (see for example Martimort (199x)). Here by contrast, we describes rules that keep the delegated decision informative. In that sense, our work is to find how to reduce the agent's discretion and preserve the incentives to signal the information through his decision. Our work is related to Armstrong (1994) ${ }^{20}$.

If we turn back to our examples of the introduction, a rule can be: in the case of political decision, a restrictions on the set of possible decision such as those who respect a budget constraint or those who favors employment,.. In vertical relations, a rule is for example a technical description of the component or a limited budget paid by the client to the supplier for R\&D.

Constraining the choice of the agent may be done in a variety of ways. We will restrict our attention to rules that are formed of a connected subset of possible decisions $d_{1}$.

\footnotetext{
${ }^{20}$ Armstrong's main results is to show that the discretion of the agent is reduced when there is a greater risk of (the agent and the principal) having diverging interest.
} 
Assumption $1 A$ rule is a connected subset $L$ of the possible decisions $d_{1}$.

Choosing a rule for the principal is to choose the boundaries $\underline{l}$ and $\bar{l}$ of the subset $L=[\underline{l}, \bar{l}]$.

To do the analysis, we have to assume that the principal can enforce the rule. i.e. She can effectively constraint the choice of the agent ${ }^{21}$. Another important assumption, that follows directly from our contractual restrictions, is that the rule cannot be state contingent. In other words, the subset $L$ is independent of $\theta$.

The optimal rule depends on how the agent acts when he receives control right over $d_{1}$. To compute it, we distinguish two cases: the case of costless signals where both incentive constraints are slack and the agent implements his preferred decision in both states of the world ${ }^{22}$ and the case where one incentive constraint binds (case of costly signals).

\subsection{Rule in the case of free lunch signals}

The following lemma reduces the set of possible rules:

Lemma 4 Without loss of generality, we can restrict our attention to rules of the form $[0, \bar{l}]$, with $\alpha_{1}+\theta_{1} \leq \bar{l} \leq \alpha_{1}+\theta_{2}$, when $\beta_{1}$ is smaller than $\alpha_{1}$. While when $\beta_{1}>\alpha_{1}$, we can consider, wlog, only rules of the form $[\underline{l},+\infty[$ with $\alpha_{1}+\theta_{2} \geq \underline{l} \geq \alpha_{1}+\theta_{1}$.

Proof: Appendix

As it appears from lemma $\mathrm{xx}$, the rules takes a different form if $\alpha_{1}$ is greater or smaller than $\beta_{1}$. this corresponds to the case where the agent takes a greater/ smaller decision than an informed principal would have taken. The reaction of the principal differs in the two situations: in one case, it is important to decrease the agent's decision, in the other case, it is important to increase it. In the paper, we treat the case in which the principal wants to decreases the decision of the agent (case where $\alpha_{1}>\beta_{1}$ ). The other case is symmetric and can be easily be computed with our analysis.

Reaction of the agent to the rule. The following proposition describes the decisions of the agent when the principal imposes a rule of the type described in lemma $\mathrm{xx}$ :

\footnotetext{
${ }^{21}$ But the agent has still the possibility of quitting the organization.

${ }^{22}$ This correspond to the case where $\Delta \theta \geq\left|\alpha_{2}-\beta_{2}\right|$.
} 
Proposition 8 When the rule is $[0, \bar{l}]$, the decisions of the agent in state $\theta_{1}$ and $\theta_{2}$ are given by:

$$
\begin{array}{rr}
d_{1}\left(\theta_{1}\right)=\alpha_{1}+\theta_{1} & \text { if } l \geq \tilde{l} \\
d_{1}\left(\theta_{1}\right)=\alpha_{1}+\theta_{1}-\sqrt{H(l)} & \text { if } l \leq \tilde{l} \\
d_{1}\left(\theta_{2}\right)=\bar{l} &
\end{array}
$$

Where $\tilde{l}=\alpha_{1}+\theta_{2}-\sqrt{2} \sqrt{\Delta \theta} \sqrt{\alpha_{2}-\beta_{2}+\Delta \theta}$ and $H(l)=\left(\alpha_{1}-l\right)^{2}-2 l \theta_{2}+$ $2\left(\alpha_{2} \theta_{1}+\beta_{2} \Delta \theta+\alpha_{1} \theta_{2}+\beta_{2} \theta_{2}+\theta_{2} \theta_{1}\right)-\theta_{1}^{2} \quad$ And $H(l)$ is a monotone and increasing function with $H(\tilde{l})=0$.

proof: appendix

In state $\theta_{2}$, the agent selects the decision that is closest to his ideal point within the allowed subset. This decision is the upper bound of the subset $L$. In state $\theta_{1}$, the agent selects the decision gives him the highest utility and such that $I C \prime_{1}$ is satisfied. This decision is either $\alpha_{1}+\theta_{1}$ or given by the constraint.

The value $\tilde{l}$ is the smallest value of $\bar{l}$ that keeps the decisions $d_{1}\left(\theta_{1}\right)=$ $\alpha_{1}+\theta_{1}, d_{1}\left(\theta_{2}\right)=\bar{l}$ incentive compatible ${ }^{23}$.

This value $\tilde{l}$ is derived from $\left(I C_{1}^{\prime}\right)$. When $\bar{l}<\tilde{l}$, if the agent wants to reveal his information in state $\theta_{1}$, his decision is not is preferred one.

limits to the imposition of rules (I): participation constraints As the rule push down the decision of the agent in at least one state of the world, we have to check that the constrained decision leaves a positive utility to the agent in both states of the world $\theta_{1}$ and $\theta_{2}$. The agent's participation constraint limits the possibilities of restricting the agent's discretion.

Lemma 5 The agent stays in the organization in both states of the world if the rule $\bar{l}$ is such that:

$$
\begin{gathered}
H(\bar{l}) \leq\left(\alpha_{1}+\theta_{1}\right)^{2}+2 \alpha_{2}\left(\beta_{2}+\theta_{1}\right)-\beta_{2}^{2}-\theta_{1}^{2} \\
\bar{l} \geq \alpha_{1}+\theta_{2}-\sqrt{\left(\alpha_{1}+\theta_{2}\right)^{2}+2 \alpha_{2}\left(\beta_{2}+\theta_{2}\right)-\beta_{2}^{2}-\theta_{2}^{2}}
\end{gathered}
$$

proof: appendix

As we said in the introduction, by paying an unconditional wage $W$, the principal increases $U^{A}$ and can solve the participation problem ${ }^{24}$. If the

\footnotetext{
${ }^{23}$ Actually, it is the only intuitive separating equilibrium.

${ }^{24}$ At least when the organization is ex ante socially efficient: $E U^{P}+U^{A} \geq 0$.
} 
principal pays an additional wage to the agent, the participation constraint are modified:

$$
\begin{gathered}
H(\bar{l}) \leq\left(\alpha_{1}+\theta_{1}\right)^{2}+2 \alpha_{2}\left(\beta_{2}+\theta_{1}\right)-\beta_{2}^{2}-\theta_{1}^{2}+W \\
\bar{l} \geq \alpha_{1}+\theta_{2}-\sqrt{\left(\alpha_{1}+\theta_{2}\right)^{2}+2 \alpha_{2}\left(\beta_{2}+\theta_{2}\right)-\beta_{2}^{2}-\theta_{2}^{2}+W}
\end{gathered}
$$

We call $\bar{l}^{I R_{1}}$ and $\bar{l}^{I R_{2}}$ the highest values of $\bar{l}$ such that $(\mathrm{xx})$ and $(\mathrm{xx})$ are satisfied.

limits to the imposition of rules (II): incentive constraint The second limit to the imposition of rules is the preservation of information transmission by the agent. As it can be seen from the demonstration of proposition $\mathrm{xx}$, we didn't use the second incentive compatible constraint $I C_{2}^{\prime}$. If this constraint isn't satisfied, it is impossible to have decisions that signals the state of the world to the principal. The rule must be set a at level that guarantee that $I C_{2}^{\prime}$ is satisfied.

Lemma 6 The rule preserves information transmission if $\bar{l}$ is such that:

$\left.\left(\alpha_{1}+\theta_{2}\right)\left(\bar{l}-\left(\alpha_{1}+\theta_{1}-\sqrt{H(\bar{l})}\right)\right)+\frac{1}{2}\left(\left(\alpha_{1}+\theta_{1}-\sqrt{H(\bar{l}}\right)\right)^{2}-\bar{l}^{2}\right) \geq \Delta \theta\left(\beta_{2}-\alpha_{2}-\frac{\Delta \theta}{2}\right)$

with $H(\bar{l})=0$ if $\bar{l} \geq \tilde{l}$.

We call $\bar{l}^{I C_{2}^{\prime}}$ the highest value of $\bar{l}$ such that $I C / 2$ is satisfied.

The optimal rule We can now compute the optimal rule that preserves agent's participation and information transmission. We identify the optimal rule with its upper bound $\overline{l^{*}}$. The optimal rule can have three possible forms:

RULE A: chooses $\overline{l^{*}}=\beta_{1}+\theta_{2} \geq \tilde{l}$.

With rule $\mathrm{A}$, the agent selects the principal's optimal decision in state $\theta_{2}$ and $\alpha_{1}+\theta_{1}$ in $\theta_{1}$. With this rule, the cost of delegation is $v_{1} \frac{\left(\alpha_{1}-\beta_{1}\right)^{2}}{2}$.

RULE B: choose $\overline{l^{*}} \leq \tilde{l}$ such that:

$$
v_{1}\left[\beta_{1}-\alpha_{1}-\Delta \theta+\sqrt{H\left(\overline{l^{*}}\right)}\right] \frac{H^{\prime}\left(\overline{l^{*}}\right)}{2 \sqrt{H\left(\overline{l^{*}}\right)}}=-v_{2}\left(\beta_{1}+\theta_{2}-\overline{l^{*}}\right)
$$

In this case, the rule is such that the principal maximizes her expected utility for $\overline{l^{*}}$, taking into account the fact that the agent reacts to the rule according to proposition 9 . 
RULE C: Choose $\overline{l^{*}}=\operatorname{Max}\left[\alpha_{1}+\theta_{1}, \bar{l}^{I C_{2}^{\prime}}, \bar{l}^{I R_{1}}, \bar{l}^{I R_{2}}\right]$.

With this rule, the principal selects the highest possible rule that preserves participation and information transmission.

Proposition 9 The optimal rule is such $\overline{l^{*}}$ that:

- If $\tilde{l} \geq \beta_{1}+\theta_{2}$ use rule $B$ if $\overline{l^{*}} \geq \operatorname{Max}\left[\alpha_{1}+\theta_{1}, \bar{l}^{I C_{2}^{\prime}}, \bar{l}^{I R_{1}}, \bar{l}^{I R_{2}}\right]$ otherwise use rule $C$.

- If $\tilde{l} \leq \beta_{1}+\theta_{2}$ use rule $A$ if $\beta_{1}+\theta_{2} \geq \operatorname{Max}\left[\alpha_{1}+\theta_{1}, \bar{l}^{I C_{2}^{\prime}}, \bar{l}^{I R_{1}}, \bar{l}^{I R_{2}}\right]$ and rule $A$ gives a greater utility than rule $B$, otherwise do as in the previous case.

The existence of two possible rule in the case $\tilde{l} \leq \beta_{1}+\theta_{2}$ is explained by the fact that the profit is not monotone on $\left[\tilde{l}, \beta_{1}+\theta_{2}\right]$.

In any cases, restricting the discretion of the agent decreases the costs of delegation.

Corrolary 2 Even if the principal can reduce agent's discretion, the costs of delegation are positive.

Add some stuff here

\section{Conclusion}

The main message of this paper is to show that when contracts à la Baron Myerson are prohibited, the principal can still extract information from the agent, either by using message games or by delegating the choice of the first project to the agent. Using the properties of signalling games, we have shown that delegation is an alternative to contracting. If delegation has the advantage of extracting agent's information, it has also some costs (loss of control). So we have shown that the principal will not always use this delegation-revelation mechanism, especially if the agent's information has little value ( $\Delta \theta$ is small) or if the divergence of interest $\left(\left|\alpha_{1}-\beta_{1}\right|\right)$ is large.

The main difference between the standard contracting technics and the model developed in this paper is that the principal cannot control the rents she pays to the agent. In the complete contract framework, rents are function of the agent's report of his private information and the principal can elicit information by paying higher rents to efficient agents. In our incomplete contract framework, the rents paid by the principal are unconditional 
on the type. The rents received by the agent is the utility he has when he is in charge of the first decision. The unconditionality of the rents increases the costs of information for the principal. We have shown that the principal can reduce the costs of delegation (and therefore agent's rents) by reducing his discretion but she cannot completely suppress these loss of control.

Pertinence of incomplete contract hypothesis: Even if our contracting restrictions seems to be strong in our paper, we believe that the incomplete contract hypothesis is a useful tool to understand how decisions are effectively taken within organizations.

day to day relations between managers and employee

extension to a continuum of decision.

\section{References}

[1] Aghion, P. and J. Tirole (1997) Formal and Real Authority in Organizations, Journal of Political Economy 105(1):1-29

[2] Armstrong, M. (1994) Delegation and Discretion, Southampton University Discussion paper 9421, Southampton UK.

[3] Baron, D. and R. Myerson (1982) Regulating a Monopolist with Unknown Costs, Econometrica 50(4), 911-930

[4] Cho I. K. and D. Kreps (1987) Signaling Games and Stable Equilibria, Quarterly Journal of Economics, 12(2):179-221

[5] Crawford, V. and J. Sobel (1982) Strategic Information Transmission, Econometrica $\mathrm{x}(\mathrm{x}): 1431-1452$

[6] Dessein W. (1999) Authority and Communication, miméo ECARE, Brussels

[7] Felli L. Preventing Collusion through Discretion, miméo LSE, London

[8] Gautier A. and D. Paolini (2000) Delegation and Reputation, miméo UCL, Louvain-la-Neuve, Belgium

[9] Grossman and Hart (1986) The Costs and Benefits of Ownership: A Theory of Vertical and Lateral Integration, Journal of Political Economy $94(x), 691-719$

[10] Hart, O. (1995) Firms, contracts and financial structure, Oxford. 
[11] Hart O. and J. Moore (1988) Incomplete Contracts and Renegotiation, Econometrica 56(x), 755-786

[12] Legros, P. (1993) Information Revelation and Repeated delegation, Games and Economic Behavior, 5(1):98-117x

[13] Martimort D. A theory of self enforceable Collusion in Organizations Rand journal of Economics

[14] Melumad N., D. Mookherjee and S. Reichelstein (1992) A Theory of Responsibility Centers, Journal of Accounting and Economics 15(x): 445-484

[15] Melumad N., D. Mookherjee and S. Reichelstein Decentralization of Incentive contracts, Rand Journal of Economics

[16] Simon, H. (1958) A Formal Theory of the Employment Relationship, Econometrica $\mathrm{x}(\mathrm{x}): 293-305$

[17] Tirole, J. (1986) Collusion and Bureaucracies

[18] Tirole, J.(1999) Incomplete Contracts: Where do we stand?, Econometrica $67(4): 741-781$ 